\title{
A CRITERION FOR THE CONFORMAL EQUIVALENCE OF A RIEMANN SPACE TO A EUCLIDEAN SPACE*
}

BY

\author{
JESSE DOUGLAS
}

\section{INTRODUCTION}

The object of the present paper is to prove the following theorem.

Theorem. Suppose that $\mathfrak{R}$, a region of a Riemann space,

$$
d s^{2}=\sum a_{i j} d x_{i} d x_{j},
$$

of $n \geqq 3$ dimensions, contains a family of curves $\mathfrak{F}$ so distributed that a unique curve of $\mathfrak{F}$ passes through each pair of points of $\mathfrak{R +}$, and that in every triangle formed by threc curves of $\mathfrak{F}$ the sum of the angles is two right angles. Then $\Re$ must be conformal to a euclidean space $\mathfrak{E}$, and in such a way that $\mathfrak{F}$ corresponds to the straight lines.

As is well known, a Riemann space of more than two dimensions is, in general, not conformally equivalent to a euclidean space. The above theorem may be regarded as furnishing a criterion for such conformal equivalence.

The theorem breaks down for the case $n=2$, not as to the conformality of the space $\Re$ to a space $\mathbb{E}$, for, of course, every surface is representable conformally on a plane, but as to the possibility of turning the family $\mathfrak{F}$ conformally into the straight lines.

Consider on any surface the isogonal trajectories of an arbitrary oneparameter family of curves. The doubly infinite totality of these trajectories

* Presented, under another title, to the Society, October 27, 1923. The results of this paper include those of a previous paper by the author, Determination of all systems of $\infty^{4}$ curves in space in which the sum of the angles of every triangle is two right angles, Bulletin of the American Mathematical Society, vol. 29 (1923), pp. 356-366.

$\uparrow$ By taking the two points infinitely close to one another, this implies that there is a unique curve of $\mathfrak{F}$ through each point in each direction. The number of curves in $\mathfrak{F}$ is necessarily $\infty^{2 n-2}$. The most usual way of defining a curve system of the type of $₹$ is by a set of differential equations of the form

$$
\frac{d^{2} x_{i}}{d s^{2}}=F_{i}\left(x_{j}, \frac{d x_{j}}{d s}\right) .
$$

We assume whatever degree of regularity in the functions $F_{i}$ is necessary to assure the function-theoretic validity of the arguments that follow. 
is termed an isogonal family. It is easily proved synthetically that in every isogonal family the sum of the angles of any triangle formed by three of the curves is two right angles, and, conversely, that this property is characteristic.* But the isogonal family is conformally equivalent to the straight lines of a plane only when the base family of $\infty^{1}$ curves is isothermal. $t$

\section{Equivalence of the family $\mathfrak{F}$ TO THE STRAight LINES BY POINT TRANSFORMATION}

Our method of proof will be entirely synthetic. For definiteness, and in order to have concrete figures to deal with, we take $n=3$, but our argument is easily extended to higher values of $n$.

First, we show that under the hypothesis of the two-right-angle property the family $\mathfrak{F}$ must be point-wise equivalent to the straight lines of a projective space.

The situation here is governed by the following fundamental theorem of projective geometry. +

Suppose that in a region of space we have a family of curves $C$ and a family of surfaces $\boldsymbol{\Sigma}$ arranged as follows:

1. There is a unique curve $C$ through any given two points and a unique surface $\Sigma$ through any given three points of the region, provided that the three points are not on the same curve $C$.

2. The curve $C$ which joins any two points of a surface lies wholly on $\Sigma$.

Then it is possible to introduce coördinates into the space so that the curves $C$ and the surfaces $\Sigma$ are represented by linear equations.

In other words, the curves $C$ and surfaces $\Sigma$ can by point transformation be converted into the straight lines and planes of a (region of a) projective space.

Our problem is, of course, to prove that the possession of the two-rightangle property by the family $\mathfrak{F}$ implies the conditions of the theorem just stated.

Each point together with a direction through it determines a curve of $\mathfrak{F}$. Consider the curves of $\mathfrak{F}$ which pass through any fixed point $O$ in the $\infty^{1}$ directions of any linear pencil. They form a surface $\Sigma$ which is easily shown to have the property that the curve $C$ of $\mathfrak{F}$ which joins anv two points of $\Sigma$ lies wholly on $\Sigma$.

* (่. S'cheffers, Isogonalkurven, Äquitangentialkurven und komplexe Zahlen, Mathematische Annalen, vol. 60 (1905), p. 504.

$\dagger$ E. Kasner, Mathematische Annalen, 1904.

$\ddagger$ See Felix Klein, Über einen Satz aus der Analysis Situs, Gesammelte Mathematische Abhandlungen, erster Band, pp. 306-310; also Über die sogenannte nicht-enklidische Geometrie in the same volume, pp. 311-343, especially pp. 333-343. 
For let $P$ and $Q$ be any two points of $\Sigma$. These must lie respectively on two curves $C_{1}$ and $C_{2}$ of $\mathfrak{F}$ which pass through $O$ in directions $d_{1}$ and $d_{2}$ belonging to the pencil defining $\Sigma$. Let $R$ be any point on the curve $C$ of $\mathfrak{F}$ which joins $P$ and $Q$, and let the curve $O R$ have at $O$ the direction $d$. Then, by applying the hypothesis of an angle sum of two right angles to the triangles $O P R, O Q R, O P Q$, we can deduce that

$$
\Varangle d_{1} d+\Varangle d d_{2}=\Varangle d_{1} d_{2} .
$$

This relation implies that $d$ belongs to the pencil of $d_{1}$ and $d_{2}$; therefore the curve $O R$ is one of those composing the surface $\Sigma$; hence the point $R$ lies on $\Sigma$. Thus the curve $C$ lies entirely on $\Sigma$, since $R$ was an arbitrary point of $C$.

It remains only to show that there is one and only one surface $\Sigma$ through any given three points not on the same curve of $\mathfrak{F}$. As defined above, a surface $\Sigma$ appears with a special point $O$ marked upon it, but this point $O$ is not really distinguished from any other point on the surface. More precisely, if $O^{\prime}$ is any point of $\Sigma$, then $\Sigma$ is identical with the locus $\Sigma^{\prime}$ of the curves $C$ of $\mathfrak{F}$ which pass through $O^{\prime}$ in the pencil of directions tangential to $\Sigma$ at $O^{\prime}$.

First, if $P$ is any point of $\Sigma$, construct the curve $C$ of $\mathfrak{F}$ which joins $O^{\prime}$ and $P$. This curve lies entirely on $\Sigma$; therefore it has at $O^{\prime}$ a direction tangential to $\Sigma$; therefore $C$ belongs to $\Sigma^{\prime}$; therefore $P$ belongs to $\Sigma^{\prime}$. Second, if $P^{\prime}$ is any point of $\Sigma^{\prime}$, it lies on a curve $C^{\prime}$ which has at $O^{\prime}$ a direction $d^{\prime}$ tangential to $\Sigma$. Allow a point $Q$ which remains always on $\Sigma$ to approach $O^{\prime}$ in the direction $d^{\prime}$. Then the curve $O^{\prime} Q$ of $\mathfrak{F}$ always lies on $\Sigma$, and evidently approaches to $C^{\prime}$ as its limit. Hence $C^{\prime}$ and therefore $P^{\prime}$ lies on $\Sigma$.

Having established the identity of $\Sigma$ and $\Sigma^{\prime}$, let us consider any three points $P, Q, R$ of space not on the same curve of $\mathfrak{F}$. Then the curves $P Q, P R$ have at $P$ two distinct directions $d_{1}, d_{2}$, which determine a pencil. Any surface $\Sigma$ containing $P, Q, R$ must contain $P$ and have the directions of the pencil $d_{1} d_{2}$ for tangential directions at $P$; these conditions, by the above, make $\Sigma$ unique.

With this we have completed our proof of the existence of a point transformation throwing $\mathfrak{F}$ in the Riemann space $\mathfrak{R}$ into the straight lines $\mathfrak{S}$ of a projective space $\mathfrak{P}$.

III. The CONES OF the ANGULAR METRIC

The measurement of angles in $\Re$ is defined by the (elemental) quadric cones

$K: \quad \sum a_{i j} d x_{i} d x_{j}=0$, 
one of which is associated with each point $O$ of the Riemann space. In fact, the angle between any two directions at $O$ is $1 / 2 i$ times the logarithm of the anharmonic ratio. of these two directions relative to the two cut from the cone associated with $O$ by the plane determined by the first two.

The projective space $\mathfrak{P}$ is metrically amorphous, having, in particular, no metric for the measurement of angles. Suppose we endow it with such a metric by allowing the point transformation which converts $\mathfrak{F}$ into the straight lines to act also on the cones $K$ of $\Re$. Then each of these goes over into a cone of elements through a point of the space $\mathfrak{P}$, and if we imagine each element extended into a complete straight line, we have a complete quadric cone associated with each point of $\mathfrak{P}$. We then define angles in $\mathfrak{P}$, as in $\mathfrak{R}$, by the logarithm of an anharmonic ratio.

We denote by $\mathfrak{P}^{\prime}$ the space $\mathfrak{P}$ as provided with its angular metric.* Since every point transformation is linear im kleinen, and linear transformations preserve anharmonic ratios, the transformation from $\Re$ to $\mathfrak{P}^{\prime}$ is angle conserving. And since the transformation throws $\mathfrak{F}$ into the straight lines of $\mathfrak{P}^{\prime}$, the sum of the angles of any rectilinear triangle in $\mathfrak{P}$ must be two right angles.

The purpose of the next two sections is to show that this implies that the cones $K$ of $\mathfrak{P}^{\prime}$ pass through a fixed conic $\boldsymbol{r}$.

In order to avoid interrupting the main thread of our argument to dispose of possible exceptions analogous to the minimal lines and planes of ordinary space, we presume the quadratic form of $\Re$ to be positive definite, so that the cones $K$ are nullpartite (have no real points except the vertex). This restriction is quite unessential, our conclusion being just as valid for indefinite quadratic forms.

\section{The case of the Plane}

We have a configuration consisting of the straight lines $\mathfrak{S}$ and the cones $K$ of the space $\mathfrak{P}^{\prime}$. Imagine that we take a section of this configuration by an arbitrary plane $\pi$. Then we obtain in $\pi$, first, its straight lines, and, second, associated with point $P$ of $\pi$ a pair of (conjugate imaginary) lines through $P$ by means of which angles at $P$ are to be measured.

Our goal in this section is to prove that the two-right-angle condition for the rectilinear triangle of $\pi$ implies that the two lines associated with an arbitrary point of $\pi$ pass respectively through two fixed points $I$ and $J$ ("circular points at infinity").

In doing this the following lemma will be very useful.

\footnotetext{
* Distance is not defined in $\mathfrak{P}^{\prime}$.
} 
Lemma. If each of three points $A, B, C$ sends its lines through two fixed points $I$ and $J$, respectively, then the same must be true of an arbitrary point of their plane.

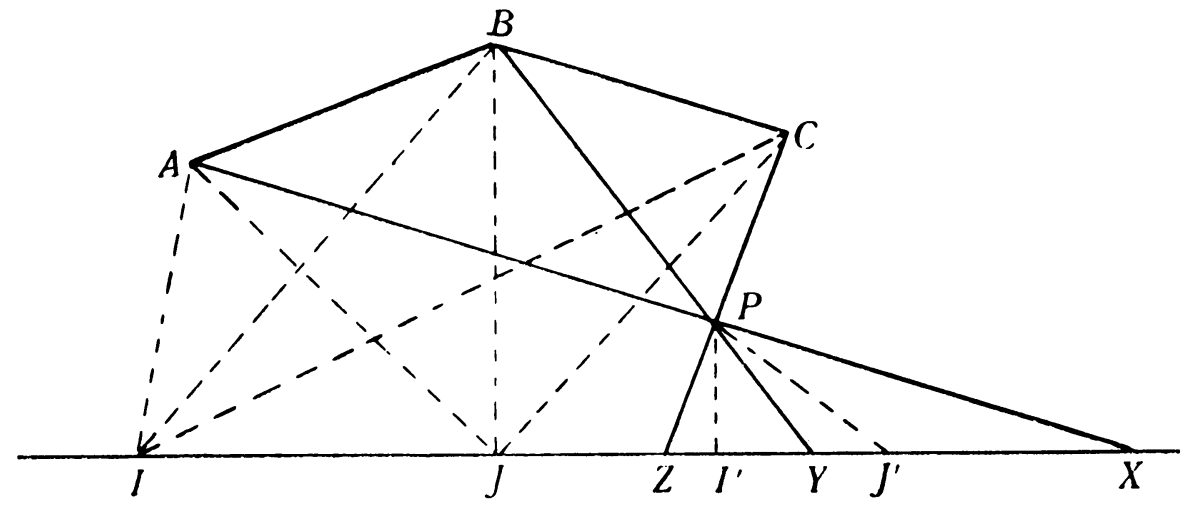

Fig. 1

Proof. Let $P$ be an arbitrary fourth point in the plane of $A, B, C$, and let the line pair of $P$ intersect the (real) line $\lambda$ of $I, J$ in $I^{\prime}, J^{\prime}$; also let $A P, B P, C P$ intersect $\lambda$ in $X, Y, Z$ respectively. Then it is easy to show that the condition of an angle sum of two right angles for the triangle $A B P$ expresses itself as follows in terms of anharmonic ratios:

$$
(X Y I J)=\left(X Y I^{\prime} J^{\prime}\right)
$$

The triangle $B C P$ gives

$$
(Y Z I J)=\left(Y Z I^{\prime} J^{\prime}\right),
$$

and from this and the preceding follows

$$
(X Z I J)=\left(X Z I^{\prime} J^{\prime}\right),
$$

which can also be obtained directly from the triangle $A C P$. These three relations imply that the point pair $I J$ is identical with $I^{\prime} J^{\prime}$, which is what was to be proved.

Our argument is not at all invalidated if $A, B, C$ are collinear. The above proof still holds for every point $P$ not on the line $A B C$, and we can then extend it to any point of this line by using any three of the points $P$ (or by continuity).

This lemma established, the next step is to cause to correspond to each point pair $A B$ a certain (real) line $\lambda$, as follows. The line pairs of $A$ and $B$ form a complete quadrilateral having two diagonals besides $A B$ (both real). 
Suppose we select one of these for a particular point pair; then the selection is determined by continuity for every point pair, at least within a certain region of the continuum of point pairs.

Now there are $\infty^{4}$ point pairs and only $\infty^{2}$ straight lines in the plane $\pi$. Therefore there are just these three possibilities: (1) the $\infty^{4}$ point pairs correspond in sets of $\infty^{2}$ to $\infty^{2}$ straight lines, or (2) in sets of $\infty^{3}$ to $\infty^{1}$ straight lines, or (3) all the $\infty^{4}$ correspond to one line.* In any case there certainly exists a line $\lambda$ together with $\infty^{\overline{2}}$ point pairs $A B$ whose associated line pairs intersect in two points $I, J$ of $\lambda$.

Let $A B$ and $A_{1} B_{1}$ be any two of the $\infty^{2}$ point pairs associated with $\lambda$, and let $A, B$ send their line pairs through the points $I, J$ of $\lambda$ while $A_{1}, B_{1}$ send theirs through $I_{1}, J_{1}$ of $\lambda$. Extend $A A_{1}, A B_{1}, B A_{1}, B B_{1}$ to meet $\lambda$ in $X, Y, Z, W$ respectively.

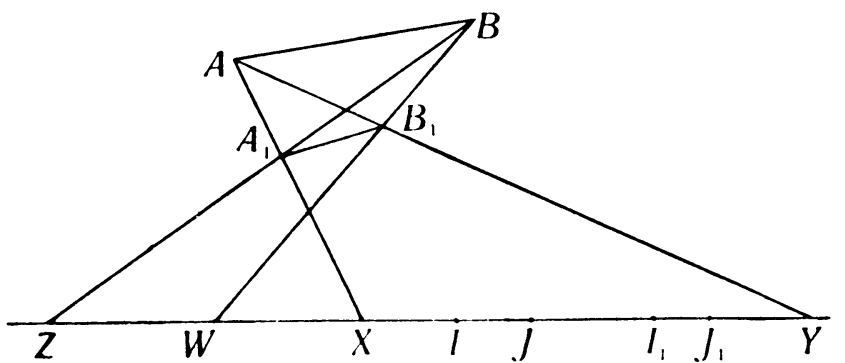

Fig. 2

Applying the two-right-angle condition to the triangles $A B A_{1}, A B B_{1}$, $A A_{1} B_{1}, B A_{1} B_{1}$, we find that the point pairs $I J$ and $I_{1} J_{1}$ must have equal anharmonic ratios with respect to any two of the four points $X, Y, Z, W$. The consequence of this is that $I_{1} J_{1}$ is identical with $I J$. We thus have four points sending their lines through $I$ and $J$, which by our lemma is enough to insure the same condition for every puint of the plane.

The above reasoning breaks down only when the line of $A_{1} B_{1}$ is identical with the line of $A B$, in which case the four points $X, Y, Z, W$ reduce to one point.t

But if this is true for every two of the $\infty^{2}$ point pairs associated with $\lambda$, these $\infty^{2}$ point pairs must be the totality of point pairs of a fixed line $\mu$, or some two-dimensional portion of that totality. Then there must be two of them such as $A B$ and $A C$, having a point $A$ in common, so that the three points $A, B, C$ have their lines going through the same two points $I, J$

* A finite number different from one is ruled out by the above lemma, also by continuity.

$\dagger$ It does not break down in a case such as where the line $A B$ contains $A_{1}$, when the four points $X, Y, Z, W$ reduce to three. 
of $\lambda$, from which the result we wish to establish follows no less than before.

\section{THE CONIC $\boldsymbol{\Gamma}$}

Consider any line $l$ of space. We begin in this section by proving that the cones associated with the different points of $l$ all pass through the same conic $\Gamma_{l} .^{*}$

Any plane $\pi$ through $l$ cuts from the $\infty^{1}$ cones related to $l \infty^{1}$ (conjugate imaginary) line pairs, all of which pass through two fixed points $I, J$ in $\pi$. Furthermore, $I, J$ are the only points common to all the line pairs and therefore the only points in $\pi$ common to all the cones.

Imagine $\pi$ to rotate about $l$. Then the points $I, J$ describe a certain locus, which is common to all the cones of $l$. Hence these $\omega^{1}$ cones all intersect in a common algebraic curve, which must be a conic, since it is cut in precisely two points by an arbitrary plane through $l$. It must be furthermore a proper conic, for the only alternative is that the cones of $l$ have a pair of generators in common, an eventuality easily disposed of.

An alternative proof is to say that the common curve of the $\infty^{1}$ quadric cones cannot be a skew quartic, for only four quadric cones can pass through a skew quartic; it cannot be a skew cubic, for such a curve is projected only from its own points by quadric cones, whereas $l$ cannot be part of the skew cubic; therefore the common curve must be a conic.

This association of a conic with each line $l$ of space produces the association of a (real) plane $\pi_{l}$, the plane of the conic, with each line $l$. $\pi_{l}$ contains the "line at infinity" $I J$ of every plane through $l . \quad \pi_{l}$ does not contain $l$, since every plane through $l$ has only two points and not a conic in common with all the cones of $l$.

Now the planes $\pi_{l}$ must in fact reduce to a single plane. For the proof, let $O$ be any point of space whose cone does not degenerate into a plane. Suppose $l$ and $m$ to be any two lines through $O$, and $n$ any third line through $O$ not coplanar with the first two. Each of the planes $\pi_{l}, \pi_{m}, \pi_{n}$ intersects, since it does not contain, its line in a unique point; let the three points of intersection be respectively $A, B, C$. Then $A, B, C$ are three distinct points. Otherwise two of them would fall together in $O$; this would cause the plane $\pi_{l}$, for instance, to contain $O$. It is easily seen that the cone of $O$ would then degenerate into the plane $\pi_{l}$, contrary to assumption.

\footnotetext{
* The only assumption made relative to $l$ is that its cones shall be proper cones, not degenerating into planes, except perhaps for isolated points on $l$. We assume, of course, that the cones do not degenerate all over space (quadratic form of $\Re$ of rank $n$ ). Then the locus of points whose cones degenerate is at most a surface, analogous to the plane at infinity in ordinary space, and we suppose $l$ not to lie on this surface.
} 
Each of the planes $\pi_{l}, \pi_{m}$ intersects the plane $l m$ in the "line at infinity" of $l \mathrm{~m}$. Therefore this line must be the line $A B$ since $\pi_{l}$ and $l m$ contain $A$ and $\pi_{m}$ and $l m$ contain $B$. Thus $\pi_{l}$ contains $B$, and by a similar argument it contains $C$. The same reasoning applies to $\pi_{m}$, so that $\pi_{l}$ and $\pi_{m}$ must fall together in the plane $A B C$.

On account of the arbitrary choice of $l$ and $m$, it follows that all the lines through $O$ determine the same plane $\Pi$. This means that the cones of any two points of a line through $O$ intersect in a conic lying in $\Pi$. Hence if $\Gamma$ is the conic in which the cone of $O$ intersects $\Pi$, the cone of every point of space must pass through $\Gamma$.

\section{REPRESENTATION ON A EUCLIDEAN SPACE}

It is evident how to bring our argument to its conclusion. We have only to introduce a euclidean space $\mathbb{F}$ and to collineate the projective angularly-metric space $\mathfrak{P}^{\prime}$ into $\mathfrak{F}$ so that the conic $\Gamma$ in $\mathfrak{B}^{\prime}$ goes over into the absolute circle (Kugelkreis) of $\mathfrak{E}$. Obviously each angle of $\mathfrak{P}^{\prime}$ is by this transformation equal to the corresponding angle of $\mathbb{E}$.

By combining the representation of $\mathfrak{R}$ on $\mathfrak{P}^{\prime}$ with that of $\mathfrak{P}^{\prime}$ on $\mathfrak{F}$, we have a transformation of $\Re$ into $\mathbb{F}$ which conserves angles and converts the family $\mathfrak{F}$ into the straight lines.

Columbia University, New YoRK, N.Y. 